\title{
Cyclic variations in distribution of the coronal green line brightness and solar magnetic field
}

\author{
Olga G. Badalyan ${ }^{1}$, V. N. Obridko ${ }^{1}$ \\ and J. Sýkora ${ }^{2}$
${ }^{1}$ Institute of Terrestrial Magnetism, Ionosphere and Radio Wave Propagation, 142190, Troitsk, Moskow region, Russia email: badalyan@izmiran.troitsk.ru
${ }^{2}$ Astronomical Institute of the Slovak Academy of Sciencies, 59060, Tatranska Lomnica, Slovak Rebublic email: sykora@ta3.sk

\begin{abstract}
Cyclic variations of the coronal green line brightness (CGLB) are investigated analysing a series of synoptic charts and a special movie assembled of them, covering 1943-2001. Regular changes of the large-scale CGLB distribution in dependence on the phase in the solar cycle are presented. A quantitative relation between the coronal green line intensity $I$ and the total magnetic field strength $B$ is derived for the period 1977-2001; the $B$ was calculated under potential approximation. A simple exponential relation of type $I \propto B^{q}$ was found to exist between the above two quantities. It is shown that the $q$ exponent may have opposite sign in the equatorial and polar zones. During phases of the solar cycle maxima the $I$ versus $B$ relation is much more complicated and cannot be expressed by such a simple formula.
\end{abstract}

\section{Introduction}

Investigation of the space-time CGLB distribution and its comparison with the magnetic field parameters seems to be quite a perspective tool when looking for different variations in solar activity and for adequate mechanism of coronal heating.

A real advantage of the CGLB measurements consists in providing a continuous numerical field characterizing level of solar activity at each point of a certain height above the Sun's photosphere. Therefore, the CGLB data favourably differ from, e.g., Wolf numbers providing only an integral characteristics for a given day, and from sunspot coordinates related to the equatorial zone and discrete regions on the solar surface. The CGLB data is at our disposal on each day of the 1943-2001 for each $5^{\circ}$ of solar latitude. All the measurements were reduced to the height of $60^{\prime \prime}$ above the solar photosphere (for more details, see Sýkora 1971, 1992).

The whole series of our CGLB data was visualized in the form of synoptic charts and a movie is composed showing evolution of the coronal structures over almost six solar cycles. The movie may be found at http://helios.izmiran.rssi.ru/hellab/Badalyan/green/. Having long series of data a comparison of the CGLB and magnetic field is possible over the solar cycles. Naturally, a close relation of them may be expected. This was earlier claimed by a number of authors (e.g., Klimchuk 2002; see also the references in Badalyan \& Obridko 2004). Nevertheless, a more detailed comparison performed at different phases in the solar cycles and for different latitudinal zones revealed that such close relation may be rather complicated and often ambiguous. 

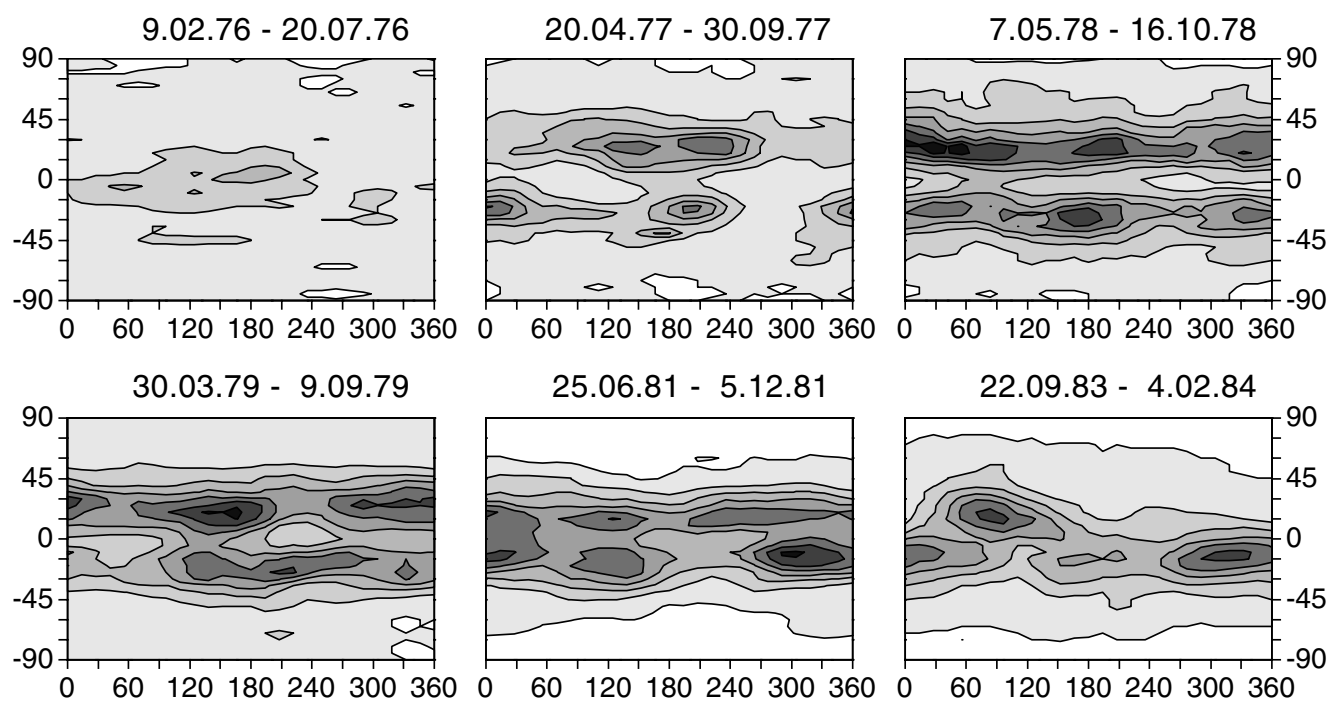

Figure 1. Characteristic synoptic charts of the coronal green line brightness for the solar cycle 21. Darker shading corresponds to greater brightness while, the white-coloured areas stand for the lowest brightness The longitude and latitude are given on the abscissa and ordinate axis, respectively. Time increases from right to left on all the synoptic charts here and below.

\section{Cyclic variations of the CGLB large-scale distribution}

The CGLB variations may be successfully described by using earlier introduced reference points of the solar cycle (Vitinsky, Kuklin \& Obridko 1986; Obridko \& Shelting 2003). It has been revealed that the large-scale CGLB distribution of the inner corona changes rapidly just close to the mentioned reference points, as they were determined from some other solar activity indices.

Basic structural peculiarities in the CGLB distribution at different phases in the 21st solar cycle are demonstrated in figure 1. Each chart of figure 1 is constructed from the data averaged over six Carrington rotations (CR). At the cycle minimum a relatively amorphous picture without distinctly manifested structures is displayed. Then, at the beginning of the ascending phase (reference point $t_{m A}$ ) at the latitudes $\pm 30^{\circ}$ the distinct bright formations arise which later, towards the end of the ascending phase $\left(t_{A M}\right)$, turn as if to two "rivers" with several brighter "islands", more or less symmetrically displaced from the equator. Subsequently, at the cycle maximum and at the beginning of the descending cycle phase $\left(t_{M D}\right)$ these two "rivers" still more approach one another, forming practically one feature during the descending phase. Finally, to the end of the descending phase $\left(t_{D m}\right)$ the united "river" disintegrates into the individual "islands" of brightening. A more detailed description of the above evolution may be found in Badalyan, Obridko \& Sýkora (2004).

The findings on the space-time CGLB distribution can be used for specifying of the solar cycle scenario. Badalyan et al.(2004) have shown that the dates of reference points deduced from the CGLB analysis agree quite well with those found by sunspots (see Obridko \& Shelting 2003). 

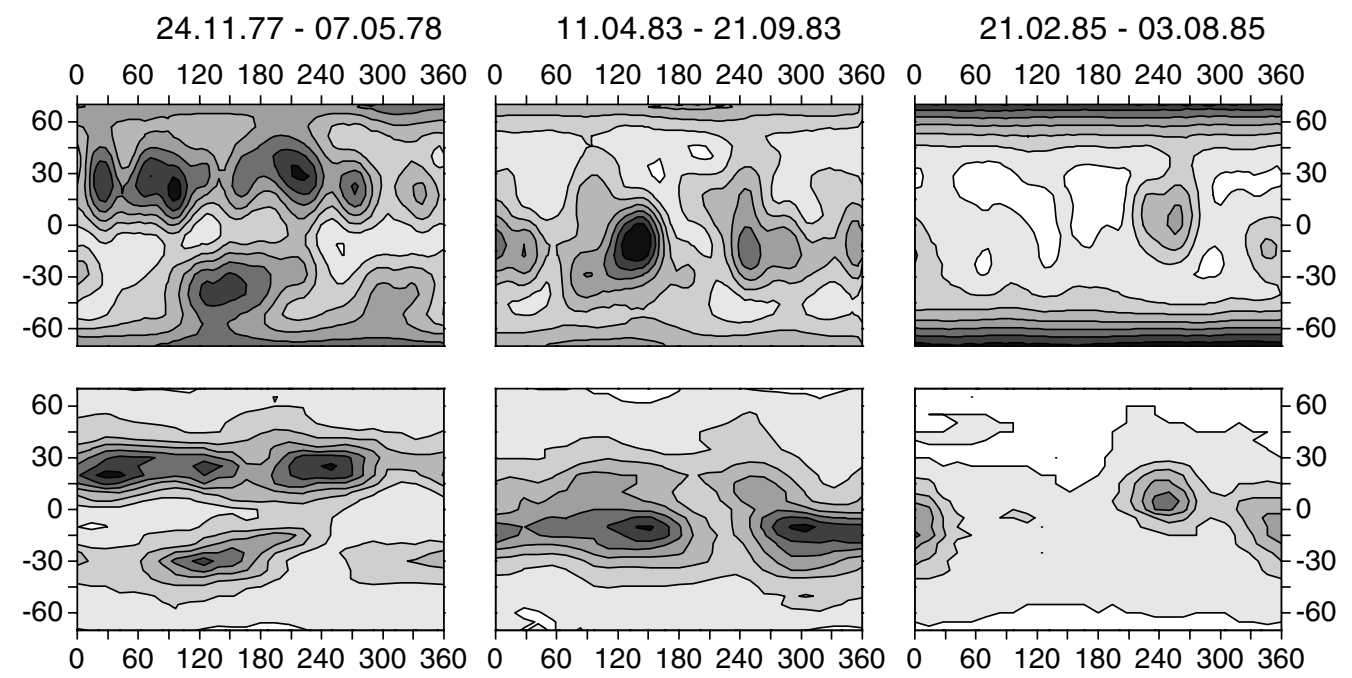

Figure 2. Comparison of three pairs of synoptic charts created for the magnetic field strength (upper panels) and the coronal green line brightness (lower panels).

\section{Comparison of the CGLB and the coronal magnetic field distributions}

Figure 2 demonstrates three pairs of the magnetic field strength and the CGLB synoptic charts for the following three periods of the 21st solar cycle: end of the ascending phase, middle of the descending phase and close to the cycle minimum. The magnetic field strength was derived for the height $1.1 R_{\odot}$ from the Wilcox Solar Observatory data and, then, averaged over six CR to be well-comparable with the CGLB data.

The comparison of both the CGLB and the coronal magnetic field strength synoptic charts was performed for the 1977-2001 period. It was revealed that the correlation between the CGLB and the magnetic field strength is rather complicated and even changes its sign at different phases of solar cycle and in different latitudinal zones (Badalyan \& Obridko 2004, Badalyan et al. 2004).

During the minimum of solar activity when the magnetic field is relatively simply organized the relation between the CGLB and the magnetic field strength may be expressed by an exponential equation of type $I \propto B^{q}$ (see, e.g., figure 3). For the zone of sunspot formation the regression displayed in figure 3 is described by the equation $I=0.31 \times B^{0.90}$. The set of points here is extracted from the synoptic chart created of the averaged data of 1783-1788 CR. It corresponds to the beginning of 1987 year, i.e., to the beginning of the ascending phase in the 22nd solar cycle. The relevant synoptic chart is similar to that at the upper left in figure 1 . For the other cases nearby and immediately after the cycle minima the coefficient $q$ is also positive and varies within 0.75-1.00.

Within the zone $40^{\circ}-70^{\circ}$ during the cycle minima the $q$ is negative. In the lower panel of figure 3 such a relation is presented for the synoptic chart averaged from 1777$1782 \mathrm{CR}$ and corresponding to 1986 year (close to minimum of the 21 st cycle). Here, the dependence is well-approximated by the equation $I=71 \times B^{-0.64}$. Here, the CGLB is extremely low. Therefore, at the lower panel the averaged values of brightness for each $5^{\circ}$ of the northern and southern hemispheric latitude are plotted, each point representing an average from six $\mathrm{CR}$ at the given latitude. For the other charts constructed from the periods of the cycles minima the $q$ exponent varies from -0.6 down to -0.8 . 

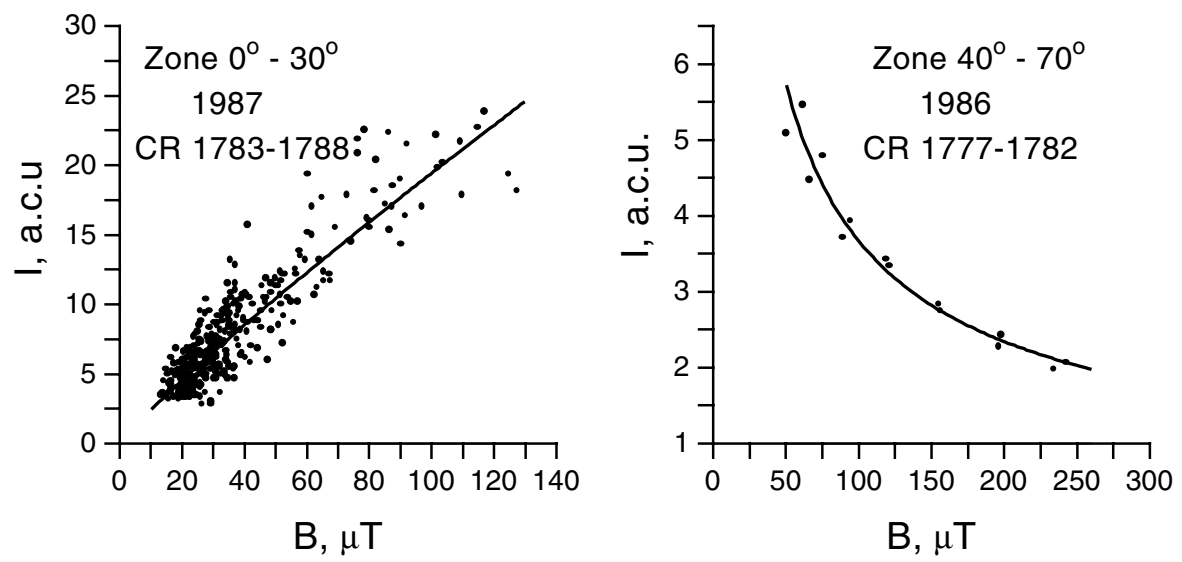

Figure 3. Relation between the coronal green line brightness and the magnetic field strength for two latitudinal zones in the period of the solar cycle minimum. The CGLB is expressed in the absolute coronal units (a.c.u.).

\section{Conclusions}

The cyclic variations of the coronal green line brightness were investigated. We have found that the substantial re-organization of the CGLB distribution takes place close to the reference points in the solar cycle. It is suggested that the data on the spacetime CGLB distribution may be well-exploited to specify a general scenario of the solar activity cycle. It was revealed that the relation between the magnetic field strength and CGLB is rather complicated and sometimes creates a negative correlation. We have found that during the minima of solar activity when the magnetic field is simply organized the dependence between the CGLB and the magnetic field strength can be described by an exponential equation of type $I \propto B^{q}$. Within the sunspot formation zone the $q$ exponent is positive and almost equal to 1.0 while, at the high latitudes, this exponent is negative, decreasing to -0.7 .

Dependence of the character of relation between the CGLB and magnetic field strength on the phase in the solar cycle seems to be an indication of different input of the fields of various scales on conditions of the green line formation and on the mechanism of coronal heating.

\section{Acknowledgements}

This work was partially supported by the Grant $02-02-16199$ of the Russian Foundation for Basic Research, by the Grant INTAS 2000-840 and by the VEGA Grant 2/4013/24 of the Slovak Academy of Sciences.

\section{References}

Badalyan, O. G. \& Obridko, V. N. 2004 Astron. Reports 81, 678-687.

Badalyan, O. G., Obridko, V. N. \& Sýkora, J. 2004 Astron. Astrophys. Transact. (in press).

Klimchuk, J. A. 2002 In Solar Coronae in the Chandra and XMM - Newton Era (eds. F. Favata \& J. J. Drake). ASP Conf. Ser. vol. 277, pp. 321-332.

Obridko, V. N. \& Shelting, B. D. 2003 Astronomy Reports 47, 953-962.

Sýkora, J. 1971 Bull. Astron. Inst. Czechosl. 22, 12-18.

Sýkora, J. 1992 Contrib. Astron. Obs. Skalnaté Pleso 22, 55-67.

Vitinsky, Yu. I., Kuklin, G. V., \& Obridko, V. N. 1986 Solnechnye dannye No. 3, 53-56. 